\title{
Harmony and Conflict of Intergenerational Relations in Rural "NEET" Families
}

\author{
Shouming $\mathrm{Li}^{1 *} \quad$ Lixia Liang ${ }^{2}$ \\ ${ }^{1,2}$ School of Political Science and Law, University of Jinan, Shandong Province, China,250022 \\ ${ }^{*}$ Corresponding author. Email: lism321@163.com
}

\begin{abstract}
The phenomenon of "NEET" in rural areas has attracted more and more attention from social. "NEET" has an important impact on intergenerational relations in families. Based on the case interviews of two types of "NEET" families in H village, the cohesion and support of family relations, the rational choice of intergenerational reciprocity, the support and cooperation under face competition, and the inheritance and continuation of family tradition are the main factors that promote the harmony of intergenerational relations of rural "NEET" families. The imbalance of rights and obligations, rational conflicts and the decline of filial piety will make the rural "NEET" families into a tense and conflict situation. Therefore, a correct understanding of the intergenerational relationship of rural "NEET" families is conducive to building a harmonious parent-child relationship.
\end{abstract}

Keywords: NEET, Intergenerational relations, Harmony and conflict

\section{THE PROPOSAL OF THE PROBLEM}

At present, about 70 percent of rural households in general agricultural areas in China have a "semi-working and semi-farming" family planning model based on intergenerational division of labor, which means that most rural youth groups are willing to become citizens. However, with the rising cost of urban life and the continuous rise of bride price in some rural areas, many scholars have questioned whether "raising children to prevent the elderly" or "raising children to eat the elderly"[1]. However, under the influence of traditional culture, Chinese parents are more willing to regard their own contribution to their children as a kind of "responsibility ethics", which is justified, and will also promote the exchange and cooperation between generations. The question I want to discuss is: What is the nature of the social problem of "NEET" behavior? What is the cause? How does this affect family intergenerational relations?

\section{LITERATURE REVIEW}

As a social phenomenon, "NEET" occurs within the scope of the family and belongs to the private sector on the surface, but the occurrence of this phenomenon and the resulting results will have many effects on society. Therefore, we can regard "NEET" as an intergenerational interaction in the family domain, whose main content is the flow of resources, but this flow is more one-way, that is, the flow of resources from parents to children. Academic circles have a lot of discussion on "NEET" and family intergenerational relations, the author will review separately.

\subsection{The Phenomenon of "NEET"}

Based on the review of existing literature, it is found that there are mainly three explanations for the phenomenon of "NEET" in rural areas. Firstly, based on the reflection of traditional Chinese culture, it is believed that parents' support and help for their children are justified and have "unlimited responsibility". Fei Xiaotong once proposed that China's intergenerational relationship belongs to the "feedback model", that is, parents provide support and help to their children when their children are young, and wait until their parents are old[2]. It is because of the existence of such cultural ethics, so that parents regardless of reward support to help children, but also for children with a more tolerant attitude.

Secondly, based on the thinking of modernization, it is believed that the acceleration of urbanization and the advent of consumer society make the rural youth bear more and more pressure, mainly in the face of the rising status of young couples in the village consumption 
standard or the high cost of living in the city, and then in the economic assistance, child care and household sharing and other aspects of the elderly parents show more or less expectation[3].

Thirdly, based on the thinking of individual education, it is believed that there are some drawbacks in both family education and school education. Some scholars believe that the root cause of the phenomenon is the delay of social weaning, the subtle influence of family on children's education, and the failure of school curriculum to adapt to the market, which will lead to the lack of personal education and the failure of their own ability and quality to meet the market requirements, so that they are more likely to become the elderly.

\subsection{The Stability and Change of Family Intergenerational Relations}

Some scholars believe that China's family intergenerational relations are generally stable. Some scholars compared the census data over the years and found that the proportion of the three-generation straight families in China has remained stable for 30 years[4]. Other scholars believe that intergenerational support still follow the "feedback model", adult children and parents in daily care, economic support, emotional comfort still exist close interaction[5].

On the other hand, many scholars believe that China's family intergenerational relations are changing with the times. Chinese traditional family intergenerational relationship is "nurturing", "father benevolent and son filial piety" is the ideal family relationship model in Chinese traditional society. It not only reflects the natural and simple relationship between parents and children, but also reflects the two-way ethical essence of "nurturing" between parents and children, which is the ethical sublimation of father and son's blood relationship[6]. But after the reform and opening up, China's family structure has changed dramatically, the family structure has continued to core, intergenerational relations have become increasingly complex[7].

\section{ANALYSIS AND DISCOVERY OF DATA}

With the normalization of "NEET" in rural families, this phenomenon is bound to have a profound impact on the intergenerational relations of families. In rural areas of China, most of the elderly do not have pension security, and the elderly themselves cannot maintain their normal daily life. Therefore, in rural "NEET" families, most of them live together with their parents and children. In this case, the maintenance of intergenerational relations requires both parents and children to get along with each other in a rational and emotional manner. Otherwise, intergenerational relations in rural "NEET" families will fall into decomposition and conflict.
$\mathrm{H}$ village is located in underdeveloped areas of central and western China, this study attempts to explain how the intergenerational relationship of rural "NEET" families is. To this end, the author analyzes six "NEET" families in the region and interviews 13 of them.

\subsection{Harmonious Intergenerational Family: Integration of Reason and Emotion}

"NEET" actually includes emotional "responsibility ethics" and rational choices based on resource flow. Therefore, the family with harmonious intergenerational relations is essentially the result of the agreement between the parents and their children on intergenerational reciprocity and emotional solidarity.

\subsubsection{Family Cohesion and Support}

Many parents take it for granted that "NEET", in the interview process, family A mother said: "We do parents is not hope that children can live better than us? We should consider everything for children in advance". It can be seen from this interview that parents regard their children's family establishment as their life obligations, especially for their sons, with the concept of "ethics of responsibility". Whether it is the cost of marriage or employment after work, parents are willing to be "nibbled".

\subsubsection{Rational Choice of Intergenerational Reciprocity}

In rural "NEET" families, parents and children often recognize this behavior, which is not only based on emotional choices, but also includes rational choices expected in the future. The mother in family $\mathrm{C}$ said: "To settle down for the child, this is not just for the child, but also for our future plans, later old, but also waiting for our young son to take care of us. " Not only the parents of family $\mathrm{C}$ mentioned that many parents of village $\mathrm{H}$ have such ideas, on the one hand, through the input of offspring resources, in order to have better development in the future; On the other hand also hope that children can develop better, in the future can provide support and help to their pension problems.

\subsubsection{Support and Collaboration in Face Competition}

With the rapid development of urbanization, most young people in rural areas choose to work and live in cities. However, young people who work and live in cities are often unable to bear high house prices and living costs. Therefore, most of them rely on parents' support and help. From this point of view, parents willing to be "nibbled" is also received local public opinion pressure and social atmosphere, only to give their children enough support and help can be recognized and accepted by the local 
villagers.

\subsubsection{Inheritance and Continuation of Family Tradition}

For families with harmonious intergenerational relations, good family style is indispensable. In the four rural families with harmonious intergenerational relations interviewed, each family has a good reputation in the village, and the people in the family will help each other to form a more united family community, which also lays the foundation for parents to provide support and help to their children. Therefore, the inheritance and continuation of good family tradition is of great significance to family intergenerational harmony.

\subsection{Family intergenerational Conflict: Decomposition of Reason and Filial Piety}

The essence of "NEET" families with intergenerational conflicts is that there is no value integration within the family, which is mainly the decomposition of rationality and filial piety, including two aspects: one is the imbalance between rights and obligations; The second is the rational conflict and the decline of filial piety.

\subsubsection{Imbalances Between Rights and Obligations}

The concept of "responsibility ethics" is prevalent in rural areas of China, that is, parents should be responsible for the future development of their children. But at the same time, many young people are showing a more selfish attitude, that is, knowing only to ask for unknowing returns. Father E of the family said: "My three sons I have arranged work and have become home, but they still think about my pension every month, I have to help do housework, or daughter-in-law looks bad." It can be seen from the interview data that in families with family intergenerational relations conflict, parents do not take it for granted and refuse to pay back to their children, which also makes the parents feel resentful and leads to tension in family intergenerational relations.

\subsubsection{Rational Conflict and the Decline of Filial Piety}

In the process of requesting from their parents, young people in rural areas lack the consciousness of independence and self-reliance. Young people often show calculation and more pursuit of their own interests, which will contradict their parents desire for their children's pension. From the interview data, it can be seen that the decline of filial piety and the unrewarded behavior of children will lead to "resentment" of parents, which leads to intergenerational conflict.

\section{CONCLUSION}

As a kind of contradictory intentional intergenerational relationship under the influence of individualized process, "NEET" reflects the resilience and internal tension of Chinese family parent-child culture in the context of social transformation[8]. The survey data show that the cohesion and support of family affection, the rational choice of intergenerational reciprocity, the support and cooperation under face competition, and the inheritance and continuation of family tradition are the main factors to promote the harmony of intergenerational relations of rural "NEET" families. The imbalance of rights and obligations, rational conflicts and the decline of filial piety will make the rural "NEET" families into a tense and conflict situation.

In the current context of urbanization, the phenomenon of "NEET" in rural areas will promote the communication and cooperation between generations of families, which is conducive to the harmony and stability of intergenerational relations of rural families, and thus will promote social stability and development. But at the same time, "NEET" should have a certain limit, excessive "NEET" will lead to the tension of family intergenerational relations. In addition, in the family pension advocated by filial piety culture, children should take the initiative to take responsibility and give emotional care to parents.

As for the intergenerational relationship of rural "NEET" families, in addition to what has been discussed above, there are several points worth paying attention to: firstly, the role of female children in the family can't be ignored. The phenomenon of "gnawing the elderly" in rural families is almost all male children, but the female children are playing a more and more important role in supporting the elderly. Therefore, in rural "NEET" families, female children play an important role in the intergenerational relationship. Secondly, paternal characteristics will affect the family intergenerational relationship. paternal characteristics mainly include economic status, social status, character characteristics, ideology, etc.

\section{ACKNOWLEDGMENTS}

Supported by National Social Science Foundation of China Key Project "Research on Family Care and Policy Support for the Elderly from the Perspective of Resource Endowment" (Project No.: 20ARK002)

\section{REFERENCES}

[1] Sakaiyue, Luo Rundong. "NEET" or "Retirement": the relationship between family intergenerational financial transfer and youth employment[J], Gansu Social Sciences. 2018 (06):146-154.

[2] Fei Xiaotong, The problem of old age support in the 
change of family structure - - The change of family structure in China[J], Journal of Peking University, $1983(03)$.

[3] Shuaipeng Dong, The competition of village status, the strategy of entering the city and the normalization of youth "NEET"[J], Contemporary youth research, 2019 (04):19-25+35.

[4] Wang Yuesheng, Theoretical Analysis of Intergenerational Relations in Chinese Families[J], Population Studies, 2008, (4):13-21.

[5] Yang Juhua, Li Lulu. Intergenerational interaction and family cohesion - comparative study of East Asian countries and regions[J], Sociological study, 2009 (03):26-53+243.

[6] He Xuefeng, Guo Junxia. Four dimensions of rural intergenerational relations[J], Social science, 2012 (07)69-78.

[7] Guo Yuhua, The Fair Logic and Its Changes in Intergenerational Relations - - An Analysis of Rural Endowment Events in Hebei[J], Chinese Academic, 2001 (04).

[8] Liu Wenrong, Intergenerational emotion and solidarity of families in transition - - Based on the comparison of two types of "NEET" families in Shanghai[J], Sociological research, 2016,31 (04): 145-168+245. 